\title{
Radiological evaluation of ex novo high grade glioma: velocity of diametric expansion and acceleration time study
}

\author{
Roberto Altieri1,2,3, Francesco Certo², Giuseppe La Rocca ${ }^{4}$, Antonio Melcarne ${ }^{5}$, \\ Diego Garbossa ${ }^{5}$, Alberto Bianchi ${ }^{6}$, Salvatore Crimi ${ }^{6}$, Alessandro Pluchino ${ }^{7}$, \\ Simone Peschillo ${ }^{1}$, Giuseppe M.V. Barbagallo ${ }^{1,2}$ \\ 1 Division of Neurosurgery, Department of Neurosciences, Policlinico “G. Rodolico”, University Hospital, Catania, Italy \\ ${ }^{2}$ Multidisciplinary Research Center on Brain Tumors Diagnosis and Treatment, University of Catania, Catania, Italy \\ ${ }^{3} \mathrm{PhD}$ program at Department of Neuroscience, University of Turin, Turin, Italy \\ ${ }^{4}$ Division of Neurosurgery, Catholic University School of Medicine, Rome, Italy \\ ${ }^{5}$ Department of Neuroscience, University of Turin, Turin, Italy \\ ${ }^{6}$ Department of General Surgery and Medical Surgery Specialties, University of Catania, Catania, Italy \\ ${ }^{7}$ Department of Physics and Astronomy "E. Maiorana”, University of Catania, Catania, Italy
}

Radiol Oncol 2021; 55(1): 26-34.

Received 15 July 2020

Accepted 16 October 2020

Correspondence to: Roberto Altieri, M.D., Department of Neurosurgery, Policlinico “G. Rodolico" University Hospital, Via Santa Sofia, 78, 95125 Catania, Italy. Phone: +39953 781 143; E-mail: roberto.altieri.87@gmail.com

Disclosure: No potential conflicts of interest were disclosed.

Background. One of the greatest neuro-oncological concern remains the lack of knowledge about the etiopathogenesis and physiopathology of gliomas. Several studies reported a strict correlation between radiological features and biological behaviour of gliomas; in this way the velocity of diametric expansion (VDE) correlate with lower grade glioma aggressiveness. However, there are no the same strong evidences for high grade gliomas (HGG) because of the lack of several preoperative MRI.

Patients and methods. We describe a series of 4 patients affected by HGG followed from 2014 to January 2019. Two patients are male and two female; two had a pathological diagnosis of glioblastoma (GBM), one of anaplastic astrocytoma (AA) and one had a neuroradiological diagnosis of GBM. The VDE and the acceleration time (AT) was calculated for fluid attenuated inversion recovery (FLAIR) volume and for the enhancing nodule (EN). Every patients underwent sequential MRI study along a mean period of 413 days.

Results. Mean VDE evaluated on FLAIR volume was $39.91 \mathrm{~mm} /$ year. Mean percentage ratio between peak values and mean value of acceleration was $282.7 \%$. Median appearance time of EN after first MRI scan was 432 days. Mean VDE was $45.02 \mathrm{~mm} /$ year. Mean percentage ratio between peak values and mean value of acceleration was $257.52 \%$. Conclusions. To our knowledge, this is the first report on VDE and acceleration growth in HGG confirming their strong aggressiveness. In a case in which we need to repeat an MRI, time between consecutive scans should be reduced to a maximum of 15-20 days and surgery should be executed as soon as possible.

Key words: acceleration time; glioblastoma; anaplastic astrocytoma; high grade glioma; radiological growth; velocity of diametric expansion

\section{Introduction}

High grade glioma (HGG) still represent a challenge for the medical community worldwide..$^{1-4}$ Currently, average survival rates reported for ana- plastic astrocytoma (AA) and glioblastoma (GBM) are 3-4 years and $10-16$ months, respectively. ${ }^{5}$ One of the greatest concerns remains the lack of knowledge about the etiopathogenesis and pathophysiology of gliomas. The new WHO classification tried 
to explain the biological behavior of these tumors, analyzing their metabolic and genetic pathways. ${ }^{5}$ However, such classification has determined further fragmentation of our knowledge, in a time when we need a minimum common denominator to solve this terrible puzzle. ${ }^{6,7}$ In recent years, research focused on glioma neuroradiological features trying to relate these with their biological behavior. ${ }^{8-15}$ As a consequence, many preoperative information about low grade gliomas (LGG) came from analysis of velocity of diametric expansion (VDE). Pallud and Mandonnet ${ }^{16-19}$ reported a strict correlation between VDE and glioma aggressiveness: growth values under $4 \mathrm{~mm}$ between two consecutive MRI studies suggest a low invasiveness rate and thus lower malignancy. ${ }^{20,21}$ However, the same evidences are not available for HGG because of the lack of several preoperative MRI and no growth patterns have been standardized for HGG. We report a series of four patients in whom HGG growth and evolution were radiologically analyzed thanks to a long preoperative follow-up since tumor diagnosis until treatment.

\section{Patients and methods}

We describe a series of 4 patients followed by the first author from 2014 to January 2019. Two patients are male, two are female, two had a pathological diagnosis of GBM, one of AA and one received a neuroradiological diagnosis of GBM. Every patient was radiologically followed through almost three pre-operative MRI scans, focusing on T1 with gadolinium and T2/fluid attenuated inversion recovery (FLAIR) sequences. Tumor volume was calculated by neurosurgeons with experience in neuro-oncological field, using Horos software for MacOs for manual segmentation of $\mathrm{T} 1$ and FLAIR 3D volumetric images in each MRI scan. ${ }^{22}$ For each patient's MRI scans mean tumor diameter $(\mathrm{MTD})^{20}$ was calculated through the following formula, starting from FLAIR-altered region's and enhancing nodule's (EN) volume (V), and expressed in millimeters $(\mathrm{mm})$ :

$$
M T D=\sqrt[3]{2 * V}
$$

[eq. 1].

It represents the reverse formula of ellipsoid volume formula calculation: $\frac{\mathrm{D} 1 * \mathrm{D} 2 * \mathrm{D} 3}{2}$ assuming the three diameters as equivalent.

Variation of MTD ( $\triangle M T D)$ between two consecutive MRI scans of each patient, was calculated as follows:
$\Delta M T D=M T D_{(n+1)}-M T D_{(n) \cup} n \neq 0 \quad$ [eq. 2]

Where $\mathrm{n}$ is the ordinal number of MRI scan for each patient.

Velocity of diametric expansion (VDE) $)^{18,20}$, or MTD difference over time (days) between two consecutive MRI scans, was calculated as:

$$
\mathrm{VDE}=\frac{\Delta M T D}{\Delta T}
$$

where $\Delta \mathrm{T}$ is the number of days between two consecutive MRI scans. VDE is reported as millimeters on days $(\mathrm{mm} / \mathrm{dd})$. Assuming a linear VDE growth, VDE for each interval of time was then calculated respect to 1-solar-year, as follow:

$$
\text { Annual VDE }=\mathrm{VDE} \times 365
$$

For each patient, mean acceleration (Acc) or VDE variation over time, was calculated as follows:

$$
A C C=\frac{V D E(n+1)-V D E(n)}{\Delta T}
$$

Where $n$ is the ordinal number of MRI scan for each patient; $\Delta \mathrm{T}$ is the number of days between two consecutive MRI scans. VDE is expressed in $\mathrm{mm} /$ $\mathrm{dd}$, thus Acc results as $\mathrm{mm} / \mathrm{dd}^{2}$.

In order to evaluate variation of VDE and Acc along observation time, further analysis were conducted for any patient. In particular, we observed peak of VDE and Acc values in all patients.

About VDE, we quantified these peaks highest value of VDE /mean VDE value ratio (listed in Table 3) in every patient along observation period, expressed as percentage [eq. 6]:

$$
\text { VDE peak }=\left(\frac{v_{\max } \times n}{\sum_{i}^{n} v_{i}}\right) \times 100
$$

Where $v$ is the VDE value; $v_{\max }$ is the highest value of VDE recorded for any patient along all observation period; $n$ total number of MRI scans for each patient; $i$ the ordinal number of MRI scan.

In Acc peak analysis, we evaluated ratio between highest Acc value and mean Acc value for any patient (listed in table 4 ), expressed as percentage, as follows:

$$
\text { Acc peak }=\left(\frac{\alpha_{\max } \times n}{\sum_{i}^{n} \alpha_{i}}\right) \times 100
$$

[eq. 7].

Where $\alpha$ is the Acc value; $\alpha_{\max }$ is the highest value of Acc recorded for any patient along all observation period; $n$ total number of MRI scans for each patient, $i$ the ordinal number of MRI scan. 
Further analysis evaluated EN/FLAIR volumes ratio (EFVR) (eq. 8), EN/FLAIR MTD ratio (EFMR) and their evolution over observation time (eq. 10 and 11), as follows:

$$
\begin{aligned}
& \text { EFVR }=\frac{\operatorname{EN~volume}_{(n)}}{\text { FLAIR volume }_{(n)}} \\
& \operatorname{EFMR}=\frac{\operatorname{EN~MTD~}_{(n)}}{\operatorname{FLAIRMTD}(n)}
\end{aligned}
$$

EFVR on single interval of time between consequent MRI scan $=E F V R /\left[T_{(n+1)}-T_{(n)}\right] \quad$ [eq. 10];

EFVR on total interval of time between first and last MRI scan of each patient $=E F V R /\left[T_{\left(n_{f}\right)}-T_{\left(n_{0}\right)}\right]$ [eq. 11];

EFMR on single interval of time between consequent MRI scan $=E F M R /\left[T_{(n+1)}-T_{(n)}\right] \quad$ [eq. 12];

EFMR on total interval of time between first and last MRI scan of each patient $=E F M R /\left[T_{\left(n_{f}\right)}-T_{\left(n_{0}\right)}\right]$ [eq. 13];

Where $\mathrm{n}$ is the ordinal number of MRI scan for each patient; $\mathrm{n}_{0}$ and $\mathrm{n}_{\mathrm{f}}$ are the first and last MRI scan for each patient, respectively.

Every analysis was conducted for whole-tumor FLAIR volume and for single EN. The analysis and graphic representation were carried out with Microsoft Office Excel® software.

In addition, a literature review was performed using PubMed MEDLINE database and searching for "radiological glioma growth", "glioma radiologic follow-up", "glioma volume growth".

\section{Ethical standards}

All procedures performed in studies involving human participants were in accordance with the ethical standards of the institutional and/or national research committee and with the 1964 Helsinki declaration and its later amendments or comparable ethical standards. Informed consent was obtained from all individual participants included in the study.

\section{Results}

\section{Case 1}

A 39 y.o. male patient was under radiological follow-up since 5 years after meningioma resection. MRI scan was obtained once a year. After 5 years of negative post-operative MRI, the exam showed hyperintensity in the right hippocampal and parahippocampal areas on T2/FLAIR sequences, without enhancement after gadolinium infusion. MTD was $35.5 \mathrm{~mm}$. For the sake of a closer follow-up, a new MRI scan with spectroscopy was scheduled 3 months later. In this second MRI scan, the FLAIR hyperintense lesion MTD was $39.7 \mathrm{~mm}$, with no enhancement after gadolinium injection. Spectroscopy showed a peak in N-acetyl-aspartate (NAA), choline (Cho) and Creatinine ( $\mathrm{Cr}$ ) values. Both data suggested a likely diagnosis of ex novo HGG. Despite strong recommendation to undergo surgery, the patient delayed surgical treatment by 3 months. The new MRI scan, obtained the day before surgery for surgical planning, showed a MTD of $45 \mathrm{~mm}$ and contrast enhancement on T1-weighted sequences. The post-operative MRI showed a complete resection of the enhancing tumor. After resection, histopathologic diagnosis revealed AA, wild-type (WT) for IDH1. All values of $\triangle \mathrm{MTD}, \mathrm{VDE}$ and Acc calculated according to equations [2], [4,] [5], [6], [7] are listed in Table 1. After tumor gross total resection, the patient underwent the Stupp regimen for adjuvant treatment and died 16 months later.

\section{Case 2}

A 37 y.o. male patient, after an episode of dysarthria, underwent a neuroradiological workout. MRI images showed a left fronto-parieto-insular enhancing lesion suspicious for ex novo GBM. FLAIR and EN MTD were respectively $56.59 \mathrm{~mm}$ and 42.02 $\mathrm{mm}$. After a week, he repeated the MRI with spectroscopy showing elevation of Cho and reduction of NAA values, elevation of Cho/NAA ratio, mild elevation of lactate signal. At the same time, central necrosis was visible. A diagnosis of GBM was made. FLAIR and EN MTD were $59.83 \mathrm{cc}$ and $48 \mathrm{cc}$ respectively. VDE was $169.23 \mathrm{~mm} /$ year for FLAIR tumor volume, whilst for EN was $311.81 \mathrm{~mm} /$ year. Due to poor neurological condition and tumor location, surgery was avoided. Following further neurological deterioration, he underwent a new MRI scan 2 weeks later. FLAIR tumor MTD was 72.60 $\mathrm{mm}$, EN MTD was $52.16 \mathrm{~mm}$. At this point, chemotherapy with temozolmide (TMZ) started. About two months later, a repeat MRI study showed good response. MTD was $67.37 \mathrm{~mm}$ for FLAIR tumor volume and $43.80 \mathrm{~mm}$ for EN, in which TMZ effect was higher (Figure 1). In fact, $\triangle \mathrm{MTD}$ was $-5.22 \mathrm{~mm}$ and - 9.36 on FLAIR and EN nodule volume, respectively. Values of $\triangle \mathrm{MTD}, \mathrm{VDE}$ and Acc calculated according to equations [2], [4,] [5], [6], [7] for every 
TABLE 1. Data about patient \#1, \#2, \#3, \#4

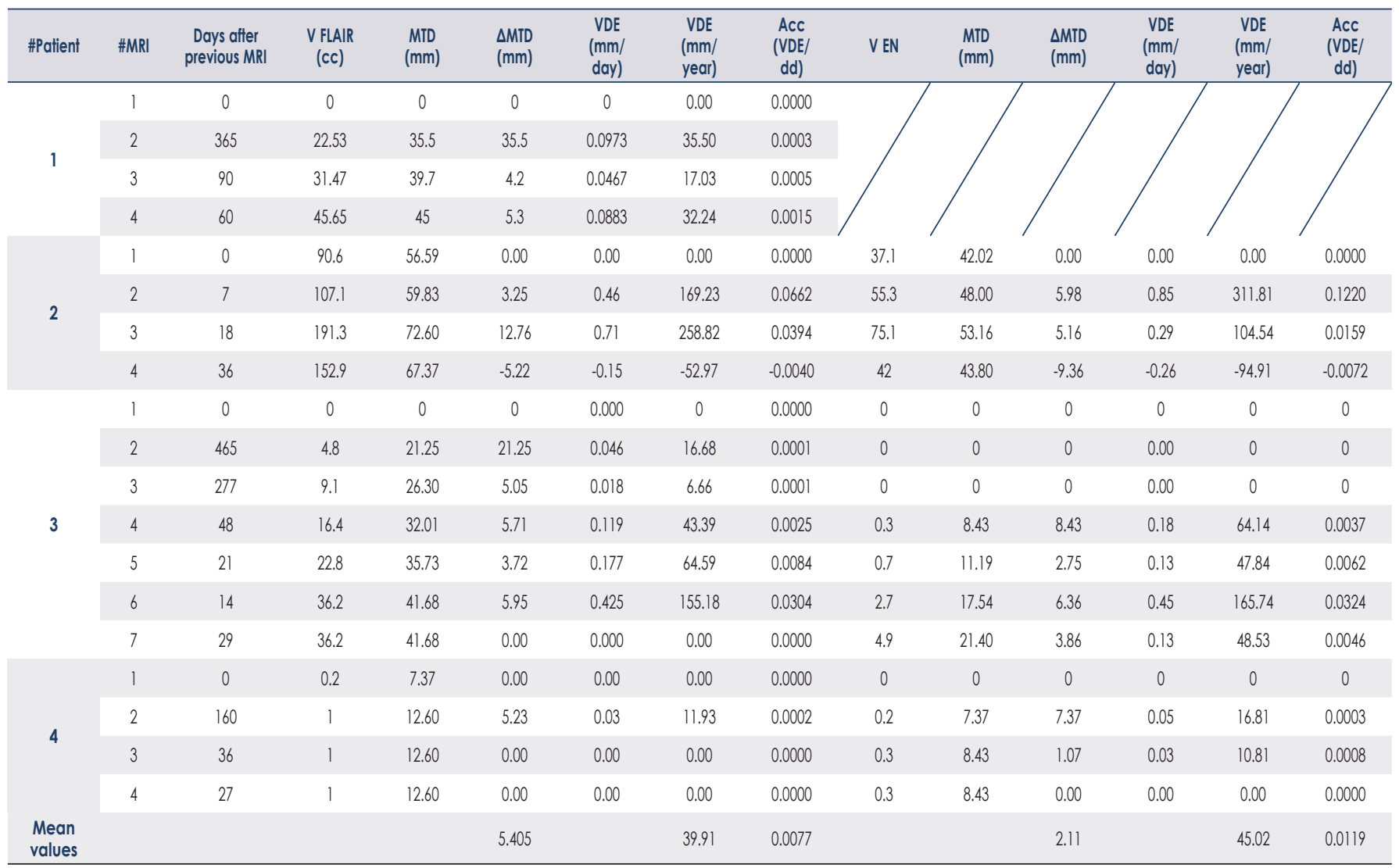

Acc = acceleration; $M R I=$ magnetic resonance imaging; $M T D=$ mean tumor diameter; $\triangle M T D=$ variation of MTD respect to previous value; $V D E=$ velocity of diameter expansion; $\mathrm{VEN}=$ volume of enhancing nodule post gadolinium; $\mathrm{V}$ FLAIR = volume of fluid attenuated inversion recovery altered regions

MRI scan are listed in Table 1, divided for FLAIR and EN volumes. 6 months after initial diagnosis the patient remains alive.

\section{Case 3}

A 72 y.o. female patient, after a seizure, underwent MRI workout without pathological findings. She repeated a MRI scan after 15 months and a FLAIR alteration in the left motor areas was seen. MTD was $21.25 \mathrm{~mm}$. She refused any treatment. MRI scan was repeated further 9 months later showing a bilateral alteration of FLAIR signal in the frontal lobes. MTD was $26.30 \mathrm{~mm}$. However, the patient chose to undergo neuroradiological followup rather than surgery. After two months, a new MRI scan showed an EN in the right frontal lobe. MTD of EN was $8.43 \mathrm{~mm}$, MTD of FLAIR altered regions was $32.01 \mathrm{~mm}$. Neurosurgeons suggested excision of the EN, but the patient refused again. Further MRI scans at 2, 6 and 10 weeks were obtained. MTD of EN were 11.19, 17.54 and $21.40 \mathrm{~mm}$, respectively. MTD of FLAIR-altered regions were $35.73,41.68$ and $41.68 \mathrm{~mm}$, respectively (Figure 2). At the end complete resection of EN was obtained; histopathological diagnosis was compatible with GBM, IDH1 WT. She underwent adjuvant therapy according to the Stupp regimen and remains alive 31 months after the index surgery. All values of $\triangle \mathrm{MTD}, \mathrm{VDE}$ and Acc calculated according to equations [2], [4,] [5], [6], [7] for every MRI scan are listed in Table 1, divided for FLAIR and EN volumes.

TABLE 2. Data on fluid attenuated inversion recovery (FLAIR) tumor and enhancing nodule (EN) volume velocity of diameter expansion (VDE). Reported as mm/year

\begin{tabular}{ccccc}
\hline \#Patient & $\begin{array}{c}\text { Mean } \\
\text { FLAIR VDE }\end{array}$ & DS FLAIR VDE & $\begin{array}{c}\text { Mean } \\
\text { EN VDE }\end{array}$ & DS EN VDE \\
\hline 1 & 21.19 & 16.26 & & \\
\hline 2 & 93.77 & 145.21 & 80.36 & 174.48 \\
\hline 3 & 40.93 & 55.88 & 46.61 & 59.19 \\
4 & 2.98 & 5.97 & 6.90 & 8.34 \\
\hline $\begin{array}{c}\text { Total mean } \\
\text { value }\end{array}$ & 39.9 & & 45.02 & \\
\hline
\end{tabular}




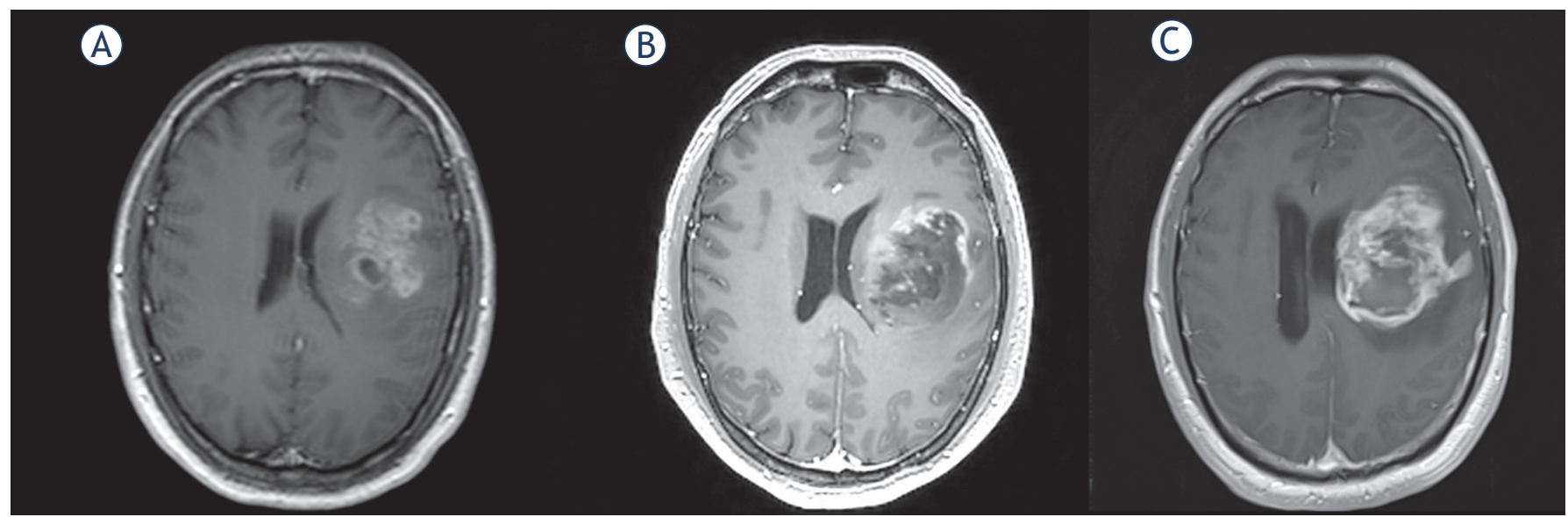

FIGURE 1. MRI scans in Patient 2 at 0 (A), 18 (B) and 54 (C) days. (TI with gadolinium sequences).

\section{Case 4}

A 56 y.o. female patient underwent MRI for persisting headache. An incidental slight alteration of FLAIR signal in the left gyrus rectus was discovered. MTD was $7.37 \mathrm{~mm}$. She repeated an MRI 5 months later to evaluate tumor evolution and an EN appeared in the previously FLAIR-altered area. MTD of the EN was $7.37 \mathrm{~mm}$, whereas MTD of FLAIR-altered area was $12.6 \mathrm{~mm}$ and there were no further changes in the next MRIs. An MRI with spectroscopy was obtained one month later and this suggested a GBM. The new MTD of the EN was $8.43 \mathrm{~mm}$. Surgical treatment was then proposed. She underwent preoperative MRI for neuronavigation one month later. MTD of the EN was 8.43 $\mathrm{mm}$ (Figure 3). Complete resection of EN was carried out and histopathological diagnosis revealed GBM IDH1 WT. She underwent adjuvant therapy with Stupp regimen and is still alive 12 months after surgery. All values of $\triangle \mathrm{MTD}, \mathrm{VDE}$ and Acc calculated according to equations [2], [4,] [5], [6], [7] for every MRI scan are listed in Table 1, divided for FLAIR and EN volumes.

\section{General evaluations}

Each patient underwent sequential MRI studies, with an average number of 4 scans along a mean period of 413 days. ENs were evident only in patient 2 on the first MRI study, whereas appeared at fourth, fourth and second MRI scan in patients n. 1, 3 and 4, respectively. No common time correlation between evidence of first FLAIR alteration and EN appearance was found. Median appearance time after first MRI scan was 432 days.

Final mean tumor volume, evaluated on each patient's last T2/FLAIR MRI sequences, was $59 \mathrm{cc}$, with a median volume variation along sequential MRIs of $6.4 \mathrm{cc}$.

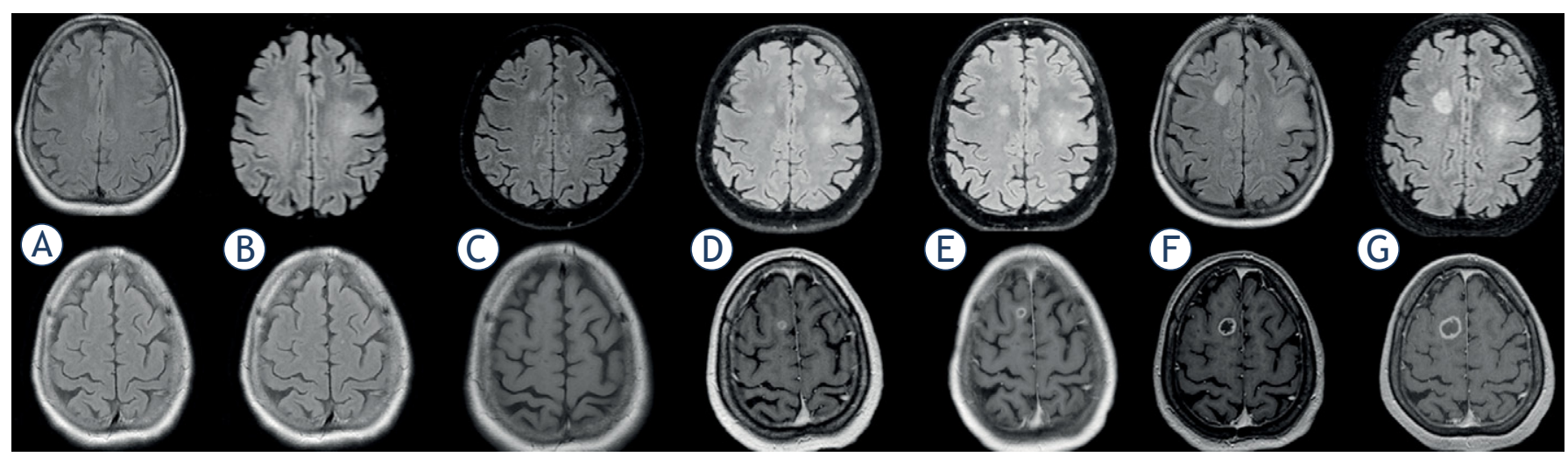

FIGURE 2. MRI scans in Patient 3 at 0 (A), 465 (B), 742 (C), 790 (D), 811 (E), 825 (F) and 854 (G) days (fluid attenuated inversion recovery [FLAIR] and T1 with gadolinium sequences). 


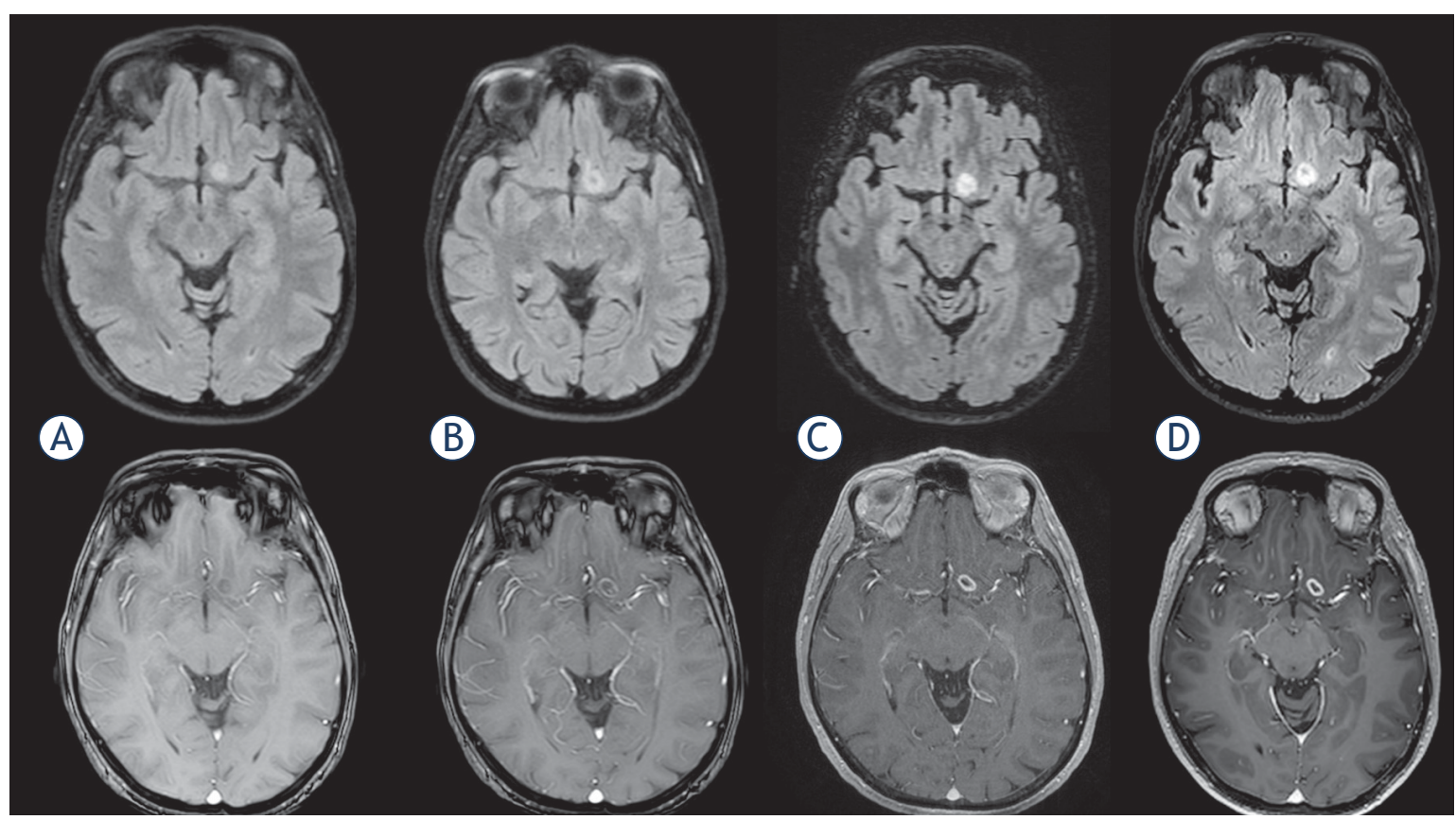

FIGURE 3. MRI scans in Patient 4 at 0 (A), 160 (B), 196 (C) and 223 (D) days (fluid attenuated inversion recovery [FLAIR] and Tl with gadolinium sequences).

Mean MTD at last MRI was 41,66 $\mathrm{mm}$ and 24.54 $\mathrm{mm}$ for FLAIR and EN volume, respectively, whilst median $\triangle$ MTD along the observation period was $5.4 \mathrm{~mm}$ and $2.11 \mathrm{~mm}$, for FLAIR and EN volume, respectively (Table 1 ).

Mean VDE was $39.9 \mathrm{~mm} /$ year and $45.2 \mathrm{~mm} /$ year for FLAIR volume and EN, respectively (Table 1, 2).

VDE analysis revealed peak in variation along time. Mean percentage maximal VDE/mean VDE ratio, calculated according to eq. 6 , was $221.49 \%$ and $259.47 \%$ for FLAIR and EN volumes, respectively (Table 3).

Acceleration analysis according to [eq. 5] revealed a mean value of $0.0077 \mathrm{~mm} / \mathrm{dd}^{2}$ and 0.0119 $\mathrm{mm} /$ days $^{2}$ for FLAIR and EN volume, respectively. Mean percentage ratio between peak values and mean value of acceleration was $282.7 \%$ for FLAIR volume and $257.52 \%$ for EN, respectively (table 4 ). In patient $n$. 1 late occurrence of the EN didn't allow complete evaluation of MTD variation, VDE and Acc (Figure 4).

Analysis of EFVR and EFMR, according to eq. 8-13 did not show significant results.

\section{Discussion}

Ex novo HGG diagnosis usually becomes evident when the tumor mass is wide and resection must be carried out as soon as possible. Thus, for each patient no many preoperative MRI scans are available for mathematical analysis of tumor growth. In the past decades, imaging studies on LGG evolution $^{23,24}$ let neurosurgeons to change their mind on treatment: from a "wait-and-see" approach to an early resection one, due to the understanding of malignant transformation risk. ${ }^{17,18}$ Indeed, Mandonnet et al. published in $2008^{19}$ a pioneering

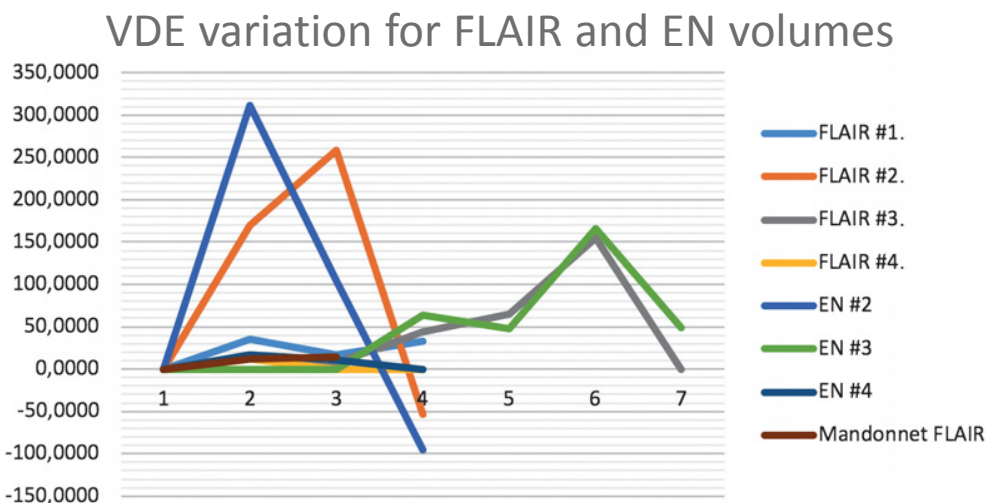

FIGURE 4. Velocity of diameter expansion (VDE) variation of mean tumor diameter (MTD) for fluid attenuated inversion recovery [FLAIR] and enhancing nodule (EN) volumes for each patient. Plus the case reported by Mandonnet et al. as specified in Discussion paragraph. Graphic shows variation of acceleration (AcC) based on VDE (mm/days $\left.{ }^{2}\right)$ for FLAIR tumor volume and EN volume along time. Except obvious similar variation for two types of volume considered, peaks are easily notable. VDE of patient \#1 is covered by patient \#4. 
TABLE 3. Raw data about velocity of diameter expansion (VDE) of fluid attenuated inversion recovery (FLAIR) and enhancing nodule (EN) volume. Mean percentage maximal VDE/mean VDE ratio could help in early high grade gliomas (HGG) diagnosis

\begin{tabular}{|c|c|c|c|c|c|c|c|c|}
\hline Interval of consecutive MRI & $\begin{array}{l}\text { VDE Pt \#1 } \\
\text { FLAIR }\end{array}$ & $\begin{array}{l}\text { VDE Pt \#2 } \\
\text { FLAIR }\end{array}$ & $\begin{array}{l}\text { VDE Pt \#2 } \\
\text { EN }\end{array}$ & $\begin{array}{l}\text { VDE Pt \#3 } \\
\text { FLAIR }\end{array}$ & $\begin{array}{l}\text { VDE Pt \#3 } \\
\text { EN }\end{array}$ & $\begin{array}{l}\text { VDE Pł } \\
\text { \#4 FLAIR }\end{array}$ & $\begin{array}{l}\text { VDE Pt \#4 } \\
\text { EN }\end{array}$ & $\begin{array}{l}\text { Mean } \\
\text { Values }\end{array}$ \\
\hline 1 & 35.50 & 169.23 & 311.81 & 16.68 & 0.00 & 11.93 & 16.81 & \\
\hline 3 & 32.24 & -52.97 & -94.91 & 43.39 & 64.14 & 0.00 & 0.00 & \\
\hline 4 & & & & 64.59 & 47.84 & & & \\
\hline 6 & & & & 0.00 & 48.53 & & & \\
\hline Mean Values & 28.26 & 125.03 & 107.15 & 47.75 & 54.37 & 3.98 & 9.21 & \\
\hline $\begin{array}{l}\text { FLAIR VDE Peak/ VDE mean } \\
\text { value ratio (percentage) }\end{array}$ & 125.63 & 135.36 & & 324.99 & & 300.00 & & 221.49 \\
\hline $\begin{array}{l}\text { EN VDE Peak/ VDE mean } \\
\text { value ratio (percentage) }\end{array}$ & & & 291.02 & & 304.81 & & 182.57 & 259.47 \\
\hline
\end{tabular}

TABLE 4. Raw data of mean acceleration (ACC) based on velocity of diameter expansion (VDE) of fluid attenuated inversion recovery (FLAIR) and enhancing nodule (EN) volume. Mean percentage maximal Acc/mean Acc ratio of each patient could help in early high grade gliomas (HGG) diagnosis

\begin{tabular}{|c|c|c|c|c|c|c|c|c|}
\hline Interval of consecutive MRI & $\begin{array}{l}\text { Acc Pt \#1 } \\
\text { FLAIR }\end{array}$ & $\begin{array}{l}\text { Acc Pt \#2 } \\
\quad \text { FLAIR }\end{array}$ & $\begin{array}{c}\text { Acc Pt \#2 } \\
\text { EN }\end{array}$ & $\begin{array}{l}\text { Acc Pt \#3 } \\
\text { FLAIR }\end{array}$ & $\begin{array}{c}\text { Acc P† \#3 } \\
\text { EN }\end{array}$ & $\begin{array}{l}\text { Acc Pt \#4 } \\
\text { FLAIR }\end{array}$ & $\begin{array}{c}\text { Acc Pt \#4 } \\
\text { EN }\end{array}$ & $\begin{array}{l}\text { Mean } \\
\text { Values }\end{array}$ \\
\hline 1 & 0.0003 & 0.0662 & 0.1220 & 0.00010 & 0 & 0.0002 & 0.0003 & \\
\hline 3 & 0.0015 & -0.0040 & -0.0072 & 0.00248 & 0.0037 & 0.0000 & 0.0000 & \\
\hline 4 & & & & 0.00843 & 0.0062 & & & \\
\hline 6 & & & & 0.00000 & 0.0046 & & & \\
\hline Mean Values & 0.0008 & 0.0339 & 0.0436 & 0.0069 & 0.0078 & 0.0001 & 0.0004 & \\
\hline $\begin{array}{l}\text { FLAIR Acc Peak/ Acc mean } \\
\text { value ratio (percentage) }\end{array}$ & 195.6695 & 195.5808 & & 439.7477 & & 300 & & 282.7495 \\
\hline $\begin{array}{l}\text { EN Acc Peak/ Acc mean } \\
\text { value ratio (percentage) }\end{array}$ & & & 280.0617 & & 414.7516 & & 77.74935 & 257.5209 \\
\hline
\end{tabular}

paper on the role of VDE in LGG and Pallud, few years later, reported that a VDE over $8 \mathrm{~mm} /$ year is an independent negative prognostic factor for survival in LGG patients..$^{20}$ In the past, few studies reported mathematical models of growth based on simple exponential growth of Volume Doubling Time (VDT) $)^{25,26}$ or linear growth of tumor radius. ${ }^{27}$ In 2015 Stensjoen et al. analyzed tumor's growth curve in 106 patients, considering two consecutive MRI scans, and reported the Gompertzian growth to be the most reliable mathematical model to predict tumor mass evolution; and this had already been reported by previous studies. ${ }^{9,28,29}$ As seen in
LGG, valuable evaluations of malignancy and evolutions could come from MTD analysis and its variation among sequential MRI studies. Mandonnet et al. reported an incidental insular tumor, followed for 9 months: VDE was 12 and $14 \mathrm{~mm} /$ year at 5 and 9 months, respectively. Histopathologic analysis then revealed an AA. ${ }^{21}$ Cochereau et al. reported a secondary GBM, in which VDE after evolution was $13 \mathrm{~mm} /$ year. ${ }^{30}$ In such cases, authors suggest that an annual VDE value over $8 \mathrm{~mm} /$ year should represent an alarm for HGG suspect. Our experience with ex novo HGG, shows even higher mean values for annual VDE: 39.91 and $45.02 \mathrm{~mm}$ /year 
for FLAIR and EN volume, respectively, enforcing previous findings and suggesting a more aggressive behaviour of an ex novo HGG respect the secondary ones. Wide differences in VDE values between our cases and cases reported by other authors, might come from different pathogenisis (primary versus secondary HGG), different observation times and biological features of the brain tumors. However, we want to underline how the VDE variate along the observation time. FLAIR VDE shows peaks, particularly in patients n. 3 and n.4, with a ratio between peak and mean VDE value of $324.99 \%(\mathrm{n}=3.25 \mathrm{~mm} / \mathrm{dd})$ and $300 \%(\mathrm{n}=3$ $\mathrm{mm} / \mathrm{dd}$ ), respectively. As shown in Figure 1, VDE variations follow a similar pattern in FLAIR and EN volume: progressive growth reach the highest value and then a rapid decrease is registered. Even if such peaks happen in different moments for each patients, such difference may be caused by different starting observation time. Speculating the possibility to follow growth curves of different HGG, all with the same onset time, peaks and following decrease could all happen in the same interval of time. Similar pattern, as in our cohort, could be found even in Mandonnet et al. case report. ${ }^{21}$ Moreover the reported mean VDE of the EN is higher than FLAIR volume VDE suggesting the higher biological aggressiveness of the enhancing regions. In addition, not yet reported to our knowledge, mean Acc values show different peaks along tumor life. In our experience mean percentage ratio between peak and mean Acc values was $270.13 \%$. Last data could improve early diagnosis of suspected primary or secondary HGG, even if a consensus about MRI timing doesn't exist. Followup MRI scans could be proposed every 4 months ${ }^{31}$ to patients with early-detected LGG. Pallud et al. proposed a timing between 6 weeks and 3 months according to Chang score. ${ }^{16}$ However, the wait and see policy in suspected HGG can let tumors grow and induce deficits. Thus, 3 months interval between two consecutive MRIs could be proposed when tumors could be misdiagnosed. Conversely, if strong suspect for HGG exists, observation could be carried out monthly. Indeed, Acc peak over $270 \%$ or VDE peak over $240 \%$ in comparison to mean values, for both FLAIR and EN volume, could help in diagnosis. Moreover, no long interval time is needed between MRIs: in our experience Acc variation could be seen even after 15-20 days. Obviously, at least three consecutive MRI scans are needed for VDE and Acc analysis. In summary, even if large series should confirm these data, we strongly propose surgical treatment in case of Acc peak and rapid VDE evolution, due to the high probability that MRI alterations refer to HGG. Such analysis could be helpful as prognostic factor and evolution parameter in case of surgical untreatable patients, i.e. due to poor general conditions or patient refusing surgery. However, sudden changes in VDE and Acc values do not have known causes. A clue could come from evolution of secondary gliomas, in which a previous LGG has been reported. By now, accumulation of mutation in LGG genes, particularly on chromatin modelling system, let a more aggressive phenotype acquisition. Maybe, as no long observation time has been obtained for HGG, our data could report a crucial moment of HGG evolution. Such peaks could refer to gain of mutation in high percentage of proliferating cells. Thus, MTD could increase in EN and FLAIR due to peripheral disposition of viable glioma cells. Such an expansion would not be compensated by core necrosis. However, too many different factors, i.e. mechanical, growth-factors-related, neo-angiogenesis, supervene in HGG infiltration and evolution. This could also explain our different results in time of peak presentation since first diagnosis, associated with the unknown time of tumor onset. It would be interesting to analyze such variation in animal models, even if inoculation of glioma cells is an important bias in infiltration pattern study. A sudden increase in MTD as reported by VDE and Acc analysis, a biopsy and genetic analysis could be investigated and related if possible.

\section{Conclusions}

To our knowledge, this is the first study describing parameters of acceleration and VDE peak in a retrospective series of ex novo HGG. Through sequential MRIs and evaluation of these parameters, an early diagnosis of HGG could be done. In addition, time between consecutive MRI scans could be reduced to 15-20 days. This work represent a pilot study on new findings of HGG growth curve, never investigated, due to short observation time in case of HGG suspect. Despite limitations in number of patients and observation time, if our data are confirmed in larger series, such values could be helpful as prognostic factors in non-surgical patient. In an attempt to obtain further confirmations, a multicenter register could be create, where all data about long-preoperative-follow-up of HGG could be reported. Moreover, studies on in-vivo and invitro models could associate genome mutations with such phenotypical variation, looking for new biological tumor pathways of interest. 


\section{References}

1. Barbagallo GM, Paratore $S$, Caltabiano R, Palmucci S, Parra HS, Privitera $G$, et al. Longterm therapy with temozolomide is a feasible option for newly diagnosed glioblastoma: a single-institution experience with as many as 101 temozolomide cycles. Neurosurg Focus 2014; 37: E4. doi: 10.3171/2014.9.FOCUS14502

2. Stupp R, Mason WP, van den Bent MJ, Weller M, Fisher B, Taphoorn MJB, et al. Radiotherapy plus concomitant and adjuvant temozolomide for glioblastoma. N Engl J Med 2005; 352: 987-96. doi: 10.1056/NEJMoa043330

3. Altieri R, Melcarne A, Soffietti R, Rudá R, Franchino F, Pellerino A, et al. Supratotal resection of glioblastoma: is less more? Surg Technol Int 2019; 35: 432-440. PMID: 31373379

4. Certo F, Stummer W, Farah JO, Freyschlag C, Visocchi M, Morrone A, et al. Supramarginal resection of glioblastoma: 5-ALA fluorescence, combined intraoperative strategies and correlation with survival. J Neurosurg Sci 2019; 63: 625-32. doi: 10.23736/\$0390-5616.19.04787-8

5. Louis DN, Ohgaki H, Wiestler OD, Cavenee WK. WHO classification of tumours of the central nervous system, Revise 4th edition. Lyon: IARC Press, 2016

6. Altieri R, Hirono S, Duffau H, Ducati A, Fontanella M, LA Rocca G, et al. Natural history of de novo high grade glioma: first description of growth parabola. J Neurosurg Sci 2020; 64: 399-403. doi: 10.23736/S03905616.17.04067-X

7. Sanai N, Alvarez-Buylla A, Berger MS. Neural stem cells and the origin of gliomas. N Engl J Med 2005; 353: 811-22. doi: 10.1056/NEJMra043666

8. Gozé $\mathrm{C}$, Blonski $\mathrm{M}$, Le Maistre $\mathrm{G}$, Bauchet L, Dezamis $\mathrm{E}$, Page $\mathrm{P}$, et al. Imaging growth and isocitrate dehydrogenase 1 mutation are independent predictors for diffuse low-grade gliomas. Neuro Oncol 2014; 16: 1100-9. doi: 10.1007/s11060-008-9680-8

9. Stensjøen AL, Solheim O, Kvistad KA, Håberg AK, Salvesen $\varnothing$, Berntsen EM. Growth dynamics of untreated glioblastomas in vivo. Neuro Oncol 2015; 17: 1402-11. doi: 10.1093/neuonc/nov029

10. Hormuth DA, Weis JA, Barnes SL, Miga MI, Rericha EC, Quaranta V, et al. A mechanically coupled reaction-diffusion model that incorporates intra-tumoural heterogeneity to predict in vivo glioma growth. J Royal Soc Interface 2017; 14: 20161010. doi: 10.1098/rsif.2016.1010

11. Raman F, Scribner E, Saut O, Wenger C, Colin T, Fathallah-Shaykh HM. Computational trials: unraveling motility phenotypes, progression patterns, and treatment options for glioblastoma multiforme. PLoS One 2016; 11 e0146617. doi: 10.1371/journal.pone.0146617

12. Batchala PP, Muttikkal TJE, Donahue JH, Patrie JT, Schiff D, Fadul CE, et al Neuroimaging-based classification algorithm for predicting $1 p / 19 q$-codeletion status in IDH -mutant lower grade gliomas. AJNR Am J Neuroradiol 2019; 40: 426-32. doi: 10.3174/ajnr.A5957

13. Altieri R, Zenga F, Ducati A, Melcarne A, Cofano F, Mammi M, et al. Tumo location and patient age predict biological signatures of high-grade gliomas. Neurosurg Rev 2018; 41: 599-604. doi: 10.1007/s10143-017-0899-8

14. Compes P, Tabouret E, Etcheverry A, Colin C, Appay R, Cordier N, et al. Neuro-radiological characteristics of adult diffuse grade II and III insular gliomas classified according to WHO 2016. J Neurooncol 2019; 142: 511-20. doi: 10.1007/s11060-019-03122-1

15. Mikkelsen VE, Stensjøen AL, Granli US, Berntsen EM, Salvesen $\varnothing$, Solheim O, et al. Angiogenesis and radiological tumor growth in patients with glioblastoma. BMC Cancer 2018; 18: 862. doi: 10.1186/s12885-018-4768-9

16. Pallud J, Taillandier L, Capelle L, Fontaine D, Peyre M, Ducray F, et al. Quantitative morphological magnetic resonance imaging follow-up of low-grade glioma: a plea for systematic measurement of growth rates. Neurosurgery 2012; 71: 729-39. doi: 10.1227/NEU.0b013e31826213de

17. Pallud J, Mandonnet E, Duffau H, Kujas M, Guillevin R, Galanaud D, et al. Prognostic value of initial magnetic resonance imaging growth rates for world health organization grade II gliomas. Ann Neurol 2006; 60: 380-3. doi: 10.1002/ana.20946

18. Mandonnet $\mathrm{E}$, Delattre J, Tanguy $M-L$, Swanson KR, Carpentier AF, Duffau $\mathrm{H}$, et al. Continuous growth of mean tumor diameter in a subset of grade II gliomas. Ann Neurol 2003; 53: 524-8. doi: 10.1002/ana.10528
19. Mandonnet E, Pallud J, Clatz O, Taillandier L, Konukoglu E, Duffau H, et al. Computational modeling of the WHO grade II glioma dynamics: principles and applications to management paradigm. Neurosurg Rev 2008; 31: 263-9. doi: 10.1007/s10143-008-0128-6

20. Pallud J, Blonski M, Mandonnet E, Audureau E, Fontaine D, Sanai N, et al. Velocity of tumor spontaneous expansion predicts long-term outcomes for diffuse low-grade gliomas. Neuro Oncol 2013; 15: 595-606. doi: 10.1093/ neuonc/nos331

21. Mandonnet E, Wait S, Choi L, Teo C. The importance of measuring the velocity of diameter expansion on MRI in upfront management of suspected WHO grade II glioma - case report. Neurochirurgie 2013; 59: 89-92. doi: 10.1016/j.neuchi.2013.02.005

22. Zeppa P, Neitzert L, Mammi M, Monticelli M, Altieri R, Castaldo M, et al. How reliable are volumetric techniques for high-grade gliomas? A comparison study of different available tools. Neurosurgery 2020; nyaa282. [Ahead of print]. doi: 10.1093/neuros/nyaa282

23. Jbabdi S, Mandonnet E, Duffau H, Capelle L, Swanson KR, Pélégrini-Issac $\mathrm{M}$, et al. Simulation of anisotropic growth of low-grade gliomas using diffusion tensor imaging. Magn Reson Med 2005; 54: 616-24. doi: 10.1002/ mrm.20625

24. Gui C, Kosteniuk SE, Lau JC, Megyesi JF. Tumor growth dynamics in seriallyimaged low-grade glioma patients. J Neurooncol 2018; 139: 167-75. doi: $10.1007 / \mathrm{s} 11060-018-2857-x$

25. Yamashita T, Kuwabara T. Estimation of rate of growth of malignant brain tumors by computed tomography scanning. Surg Neurol 1983; 20: 464-70. doi: 10.1016/0090-3019(83)90029-0

26. Schwartz M. A biomathematical approach to clinical tumor growth. Cancer 1961; 14: 1272-94. doi: 10.1002/1097-0142(196111/12)14:6<1272::aidcncr2820140618>3.0.co;2-h

27. Harpold HLP, Alvord EC, Swanson KR. The evolution of mathematical modeling of glioma proliferation and invasion. J Neuropathol Exp Neurol 2007; 66: 1-9. doi: 10.1097/nen.0b013e31802d9000

28. Laird AK. Dynamics of tumor growth. Br J Cancer 1964; 13: 490-502. doi: 10.1038/bjc.1964.55

29. Chignola R, Foroni RI. Estimating the growth kinetics of experimental tumors from as few as two determinations of tumor size: implications for clinical oncology. IEEE Transactions on Biomed Eng 2005; 52: 808-15. doi: 10.1109/TBME.2005.845219

30. Cochereau J, Herbet G, Rigau V, Duffau H. Acute progression of untreated incidental WHO Grade II glioma to glioblastoma in an asymptomatic patient. J Neurosurg 2016; 124: 141-5. doi: 10.3171/2014.12.JNS141851

31. Shah AH, Madhavan K, Heros D, Raper DMS, lorgulescu JB, Lally BE, et al. The management of incidental low-grade gliomas using magnetic resonance imaging: systematic review and optimal treatment paradigm. Neurosurg Focus 2011; 31: E12. doi: 10.3171/2011.9.FOCUS11219 